\title{
Application of the Wagner-Nelson Absorption Method to the Two-Compartment Open Model
}

\author{
John G. Wagner ${ }^{1,2}$ \\ Received Jan. 24, 1974-Final July 22, 1974
}

\begin{abstract}
This report considers the application of the Wagner-Nelson method to both one-and two-compartment open model data when there is no competing reaction at the absorption site. Equations are derived which show that application of the Wagner-Nelson method to data which obey the twocompartment open model with first-order absorption allows accurate estimation of not only the rate constant $k_{a}$ but also the parameters of the two-compartment open model, namely $k_{12}, k_{21}$, and $k_{e l}$. In the example given, this new method was more accurate than the classical "feathering" or "back-projection" method. The appropriate criterion for "collapsing" the two- to the onecompartment open model is given. In cases where the one-compartment open model applies, and absorption is first order but abruptly ceases after some time, it is shown that $k_{a}$ may be accurately estimated by application of the Guggenheim method to the $A_{T} / V$ values calculated for the absorption phase.
\end{abstract}

KEY WORDS: Wagner-Nelson method; absorption rate constant; one-compartment open model; Loo-Riegelman method; two-compartment open model; competing reactions at absorption site.

\section{INTRODUCTION}

The method of Wagner and Nelson $(1,2)$ as originally published, appeared to provide (a) plots of amount of drug absorbed per unit volume of distribution vs. time and (b) plots of percent of drug absorbed vs. time. The method was not designed or claimed to be a method to determine an "absorption rate constant." Wagner and Nelson (1) stated: "When the cumulative percentages absorbed are plotted against time, the resulting plots may contain linear segments; the slope of such a linear segment is the absorption rate in percent/hour. If the plot is curved, or contains curved or

Supported in part by Public Health Service Grant 5-P11-GM15559.

${ }^{1}$ College of Pharmacy and Upjohn Center for Clinical Pharmacology, The University of Michigan, Ann Arbor, Michigan 48104.

${ }^{2}$ Address reprint requests to Dr. John G. Wagner, Upjohn Center for Clinical Pharmacology, The University of Michigan, Ann Arbor, Michigan 48104. 
linear segments, it may often be resolved to yield the components of the rate $d \% A_{T} / d t$." Notari et al. (3), Perrier and Gibaldi (4), and Leeson and Weintraub (5) have discussed problems that arise in estimating "absorption rate constants" when the drug is absorbed and also simultaneously lost to an extravascular compartment via either a parallel first-order or a zero-order process. Loo and Riegelman (6) applied the Wagner-Nelson method (1) to data which obviously obeyed the two-compartment open model with both first-order and zero-order input to the central compartment and stated: "It appears that both the bi-exponential and the occasional appearance of maxima are artifacts of the numerical method."

The method of Loo and Riegelman (6) requires that the drug be administered intravenously before plasma concentration data obtained following oral administration may be evaluated. The method of Wagner and Nelson (1) does not require such intravenous data. Since, intravenous data are frequently not available, the use of the Wagner-Nelson method becomes attractive. It is shown that in the special case where data obey the twocompartment open model with first-order absorption (without a parallel competing reaction), application of the Wagner-Nelson method allows estimation of all the parameters of the two-compartment model.

In resolving Wagner-Nelson "absorption" plots, the original numerical values obtained by the method should be utilized. The values should not be converted to "percentage absorbed" values as originally published (1). This is important since if absorption abruptly ceases because of a "window effect" at the absorption site, the wrong asymptote would be used if the conversion to percentages were made.

\section{THEORETICAL}

\section{Relative Magnitude of Asymptotes by Wagner-Nelson (1) and Loo-Riegelman Methods}

If it is assumed that data obey the two-compartment open model, then the asymptote obtained by application of the Loo-Riegelman method (6) to the oral data is given by equation 1 :

$$
A_{\infty} / V_{1}=k_{\mathrm{el}} \int_{0}^{\infty} C_{1}(t) d t
$$

where $A_{\infty}$ is the amount of drug absorbed to infinite time (which is equal to $F D$ where $F$ is the fraction of the doses, $D$, which is absorbed), $V_{1}$ is the volume of the inner (central) compartment of the two-compartment model shown in Scheme I below, $k_{\mathrm{el}}$ is the elimination rate constant, and the integral is the 
total area under the plasma concentration-time curve (taken as representative of the area under the concentration-time curve of the central compartment).

Under the same assumptions, the asymptote obtained by application of the Wagner-Nelson method (1) to the oral data is given by equation 2:

$$
A_{\infty} / V_{d_{\text {area }}}=\beta \int_{0}^{\infty} C_{1}(t) d t
$$

In equation $2, V_{d_{\text {area }}}$ and $\beta$ are given by equations 3 and 4 , respectively:

$$
\begin{gathered}
V_{d_{\mathrm{area}}}=\left(\alpha / k_{21}\right) V_{1}=\left(k_{\mathrm{e} 1} / \beta\right) V_{1} \\
\beta=\frac{1}{2}\left\{\left(k_{12}+k_{21}+k_{\mathrm{e} 1}\right)-\left[\left(k_{12}+k_{21}+k_{\mathrm{el}}\right)^{2}-4 k_{21} k_{\mathrm{e} 1}\right]^{1 / 2}\right\}
\end{gathered}
$$

From equations 1,2 , and 3 , one obtains the relationship given in equation 5 :

$$
\frac{\text { Asymptote obtained by Loo-Riegelman method }}{\text { Asymptote obtained by Wagner-Nelson method }}=\alpha / k_{21}=k_{\mathrm{e} i} / \beta
$$

Equation 5 will hold when there is no competing reaction at the absorption site, and for all cases of the two-compartment open model i.e., when absorption is nonuniform, or when absorption obeys uniform kinetics such as first order.

It follows that if one compares two oral treatments (e.g., two different tablets or tablet vs. solution of the drug) and perforns only the WagnerNelson method, then the ratio of the asymptotes obtained by means of equation 2 is the ratio of the relative amounts of drug absorbed following the two treatments, provided that $k_{\mathrm{el}} / \beta$ remains constant for the subject.

\section{The Two-Compartment Open Model with First-Order Absorption}

If data obey this model (shown in Scheme I), then the " $K$ " in the original Wagner-Nelson paper (1) becomes equivalent to " $\beta$," as defined by equation 4 , since an estimate of " $\beta$ " is obtained from the terminal oral plasma concentration data.

The model is

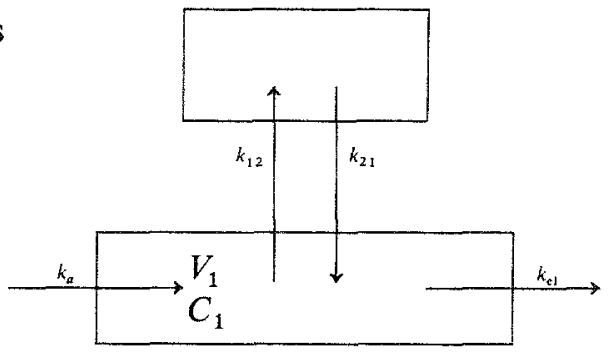

Scheme I 
For the model shown in Scheme I, $C_{1}$ is given by equation 6 , where $C_{0}=$ $\underset{\text { dose }}{\text { absorbed }} / V_{1}$.

$$
\begin{aligned}
C_{1}(t)= & k_{a} C_{0}\left[\frac{\left(k_{21}-\alpha\right)}{\left(k_{a}-\alpha\right)(\beta-\alpha)} e^{-\alpha t}+\frac{\left(k_{21}-\beta\right)}{\left(k_{a}-\beta\right)(\alpha-\beta)} e^{-\beta t}\right. \\
& \left.+\frac{\left(k_{21}-k_{a}\right)}{\left(\alpha-k_{a}\right)\left(\beta-k_{a}\right)} e^{-k_{a} t}\right]
\end{aligned}
$$

By algebraic manipulation it may be shown that

$$
\begin{aligned}
F(T)=C_{1}(T)+\beta \int_{0}^{T} C_{1}(t) d t= & C_{0}\left[\frac{k_{21}}{\alpha}+\frac{1}{\alpha\left(k_{a}-\alpha\right)}\left\{k_{a}\left(\alpha-k_{21}\right) e^{-\alpha T}\right.\right. \\
& \left.\left.-\alpha\left(k_{a}-k_{21}\right) e^{-k_{\alpha} T}\right\}\right]
\end{aligned}
$$

A plot of $F(T)$ vs. $T$ is equivalent to a "Wagner-Nelson" plot. ${ }^{3}$

The following is a method for obtaining preliminary estimates of all parameters of the model:

Let $A_{s}=$ the asymptote of the $F(T), T$ plot. Then

$$
A_{s}=\beta \int_{0}^{\infty} C_{1}(t) d t=C_{0}\left(k_{21} / \alpha\right)
$$

If $k_{a}>\alpha>\beta$, and since $\alpha>k_{21}$, equation 7 may be written ${ }^{4}$ as

$$
F(T)=A_{s}+I_{1} e^{-\alpha T}-I_{2} e^{-k_{a} T}
$$

where

$$
I_{1}=C_{0}\left\{\frac{k_{a}\left(\alpha-k_{21}\right)}{\alpha\left(k_{a}-\alpha\right)}\right\}
$$

and

$$
I_{2}=C_{0}\left\{\frac{\left(k_{a}-k_{21}\right)}{\left(k_{a}-\alpha\right)}\right\}
$$

In application of the Wagner-Nelson method, the terminal $C_{1}, t$ data are fitted to the equation

$$
C_{1}(t)=A_{2} e^{-\beta t}
$$

\footnotetext{
${ }^{3}$ Equation 7 indicates what would be obtained if a Wagner-Nelson calculation (assuming a one-compartment open model) were applied to data generated from equation 6 .

${ }^{4}$ The alternative case for $\alpha>k_{a}>\beta$ is treated in the Appendix.
} 
where

$$
A_{2}=\frac{k_{a} C_{0}\left(k_{21}-\beta\right)}{\left(k_{a}-\beta\right)(\alpha-\beta)}
$$

After combining equations 8 and 13 , one obtains

$$
k_{a}=\frac{\beta A_{2}(\alpha-\beta)}{(\alpha-\beta) A_{2}+\beta C_{0}-\alpha A_{s}}
$$

After combining equations 8 and 10 , one obtains

$$
k_{a}=\alpha I_{1} /\left(I_{1}+A_{s}-C_{0}\right)
$$

By equating the right-hand sides of equations 14 and 15 , one obtains

$$
C_{0}=\frac{\beta A_{2}(\alpha-\beta)\left(I_{1}+A_{s}\right)+\alpha I_{1}\left[\alpha A_{s}-(\alpha-\beta) A_{2}\right]}{\alpha \beta I_{1}+\beta A_{2}(\alpha-\beta)}
$$

Rearrangement of equation 8 gives

$$
k_{21}=\alpha A_{s} / C_{0}
$$

Also,

$$
\begin{aligned}
& k_{\mathrm{el}}=\alpha \beta / k_{21} \\
& k_{12}=\alpha+\beta-k_{21}-k_{\mathrm{el}}
\end{aligned}
$$

The estimates of $\alpha$ and $I_{1}$ needed to utilize equations 16-19 are obtained from the least-squares line for $\ln \left[F(T)-A_{s}\right], T$ values where the $F(T)$ values are past the peak of the $F(T), T$ plot. This line is

$$
\ln \left[F(T)-A_{s}\right]=\ln I_{1}-\alpha T
$$

which may be written as

$$
\left[F(T)-A_{s}\right]=I_{1} e^{-\alpha T}
$$

This may be seen from equation 9 where $e^{-k_{a} T} \simeq 0$. Note also that $F(T)$ is a maximum when $d F(T) / d T=0$. Differentiating equation 7 gives:

$$
\frac{d F(T)}{d T}=-\frac{k_{a}\left(\alpha-k_{21}\right)}{\left(k_{a}-\alpha\right)} e^{-\alpha T}+\frac{k_{a}\left(k_{a}-k_{21}\right)}{\left(k_{a}-\alpha\right)} e^{-k_{a} T}
$$

When $d F(T) / d T=0$, then

$$
\frac{k_{a}\left(\alpha-k_{21}\right)}{\left(k_{a}-\alpha\right)} e^{-\alpha T_{\max }}=\frac{k_{a}\left(k_{a}-k_{21}\right)}{\left(k_{a}-\alpha\right)} e^{-k_{a} T_{\max }}
$$


Rearrangement of equation 23 , followed by taking natural logarithms of both sides, gives

$$
T_{\max }=-\frac{1}{\left(k_{a}-\alpha\right)} \ln \left[\frac{\alpha-k_{21}}{k_{a}-k_{21}}\right]
$$

\section{Collapsing of the Two-Compartment Open Model to the One-Compartment Open Model}

For the case of bolus intravenous injection, the model shown in Scheme I has no input " $k_{a}$ step," but rather the dose, $D$, is introduced into the central compartment at $t=0$. The equation for $C_{1}$ as a function of time in this case is equation 25 :

$$
C_{1}(t)=\frac{D}{V_{1}(\alpha-\beta)}\left[\left(k_{21}-\beta\right) e^{-\beta t}-\left(k_{21}-\alpha\right) e^{-\alpha t}\right]
$$

Equation 25 may be written as equation 26 :

$$
C_{1}(t)=A e^{-\alpha t}+B e^{-\beta t}
$$

Also,

$$
C_{1}^{0}=A+B
$$

Hence,

$$
C_{1} / C_{1}^{0}=\{A /(A+B)\} e^{-\alpha t}+\{B /(A+B)\} e^{-\beta t}
$$

In the above,

Hence,

$$
\begin{aligned}
& A=\frac{D\left(\alpha-k_{21}\right)}{V_{1}(\alpha-\beta)} \\
& B=\frac{D\left(k_{21}-\beta\right)}{V_{1}(\alpha-\beta)}
\end{aligned}
$$

where

$$
B /(A+B)=\left(k_{21}-\beta\right) /(\alpha-\beta)=V_{1} / V_{d_{\text {extrap }}}
$$

$$
V_{d_{\text {extrap }}}=D / B
$$

The two-compartment open model "collapses" to the one-compartment open model as $B /(A+B) \rightarrow 1$, or as $\left(k_{21}-\beta\right) /(\alpha-\beta) \rightarrow 1$, or as $V_{1} \rightarrow V_{d_{\text {extrap }}}$. This is also clarified by writing equation 28 as equation 33 .

$$
C_{1} / C_{1}^{0}=\left(1-V_{1} / V_{d_{\text {extrap }}}\right) e^{-\alpha t}+\left(V_{1} / V_{d_{\text {extrap }}}\right) e^{-\beta t}
$$

Hence the ratio $\alpha / \beta$ is not the determining factor in "collapsing" but rather the relative magnitudes of $k_{21}, \alpha$, and $\beta$, and particularly of $k_{21}$ and $\alpha$. If 
$B /(A+B)$ has a numerical value equal to or greater than about 0.9 , then the one-compartment open model will be a reasonable approximation even though the data actually obey the two-compartment open model. The author believes that the two-compartment open model is the actual "minimum" model for linear systems in pharmacokinetics, but under the above conditions the one-compartment open model becomes a good approximation and is useful because of its greater simplicity. In the next section, it is assumed that the one-compartment open model applies; hence $K_{E}$ replaces $\beta$, since when "collapsing" occurs completely then $K_{E}=k_{\mathrm{eI}}=\beta$. It should be noted that if "collapsing" occurs, and the Wagner-Nelson method is applied to such data, $F(T)$ will not reach a maximum value and then decrease beyond the maximum, but, instead, the $F(T), T$ plot will slowly approach an asymptote.

\section{Case Where Absorption Abruptly Ceases}

Perrier and Gibaldi (4) also considered their "all or none" phenomenon, where drug is being absorbed at a first-order rate from a timed-release or other dosage form, but at some time the drug passes the absorption site and absorption abruptly ceases. They stated that "if the drug is less than fully available due to an all or none phenomenon, the percent of drug absorbed can be calculated relative to the total dose administered rather than relative to the total amount of drug eventually absorbed, $A_{\infty}$." It is shown in the Experimental and Results section that the Wagner-Nelson method does give the correct bioavailability estimate, and the correct first-order rate constant for absorption in such a case can be calculated without knowing the total amount absorbed per milliliter of the volume of distribution.

\section{EXPERIMENTAL AND RESULTS}

\section{Simulation Example No. 1 in Which Equations 6-39 Are Applied}

A simulation was performed with $k_{a}=2, \alpha=0.5, \beta=0.1, k_{21}=0.25$, and $C_{0}=100$. Using equation 6 , one obtains equation 34 for these values of the constants:

$$
C_{1}(t)=83.3333 e^{-0.5 t}+39.4737 e^{-0.1 t}+122.8070 e^{-2 t}
$$

Using equation 7 , one obtains equation 35 :

$$
F(T)=50+66.6666 e^{-0.5 T}-116.6666 e^{-2 T}
$$

Values of error-free $C_{1}, t$ and $F(T)$ obtained with equations 34 and 35 are shown in columns 2 and 3, respectively, in Table I. Values of $F(T)$ obtained by applying the Wagner-Nelson method, using the estimated $\beta(\hat{\beta})$ from the terminal $C_{1}, t$ data and the trapezoidal rule for the areas, are shown in the fifth column of Table I. 
Table I. Simulation Example No. 1

\begin{tabular}{|c|c|c|c|c|c|c|c|}
\hline & $t(\mathrm{hr})$ & $C_{1}(T)$ & $F(T)$ & $\begin{array}{l}\text { Trape- } \\
\text { zoidal } \\
\text { area }\end{array}$ & $F(T)^{a}$ & $\begin{array}{c}F(T)-A_{s} \\
=F(T)- \\
50\end{array}$ & $\begin{array}{r}\widehat{F(T)}-\widehat{A_{s}} \\
=F(T)- \\
\quad 50.234\end{array}$ \\
\hline & 0 & 0 & 0 & 0 & 0 & & \\
\hline & 0.1 & 17.804 & 17.897 & 0.8902 & 17.893 & & \\
\hline & 0.2 & 31.775 & 32.118 & 3.3692 & 32.112 & & \\
\hline & 0.4 & 50.973 & 52.160 & 11.6440 & 52.137 & & \\
\hline & 0.6 & 61.921 & 64.249 & 22.933 & 64.214 & & \\
\hline & 0.8 & 67.504 & 71.133 & 38.876 & 71.392 & & \\
\hline & 1.0 & 69.641 & 74.646 & 45.590 & 74.200 & & \\
\hline \multirow[t]{18}{*}{$T_{\max }=$} & 1.29726 & 69.063 & 76.138 & 70.206 & 76.054 & & \\
\hline & 1.4 & 68.231 & 76.011 & 77.259 & 76.957 & & \\
\hline & 1.6 & 66.075 & 75.200 & 90.689 & 75.144 & & \\
\hline & 1.8 & 63.496 & 73.917 & 103.646 & 73.861 & & \\
\hline & 2.0 & 60.726 & 72.388 & 116.069 & 72.333 & & \\
\hline & 2.4 & 55.140 & 69.119 & 139.242 & 69.064 & & \\
\hline & 2.8 & 49.929 & 66.008 & 160.256 & 65.955 & 16.008 & $15.721)$ \\
\hline & 3.2 & 45.284 & 63.266 & 179.298 & 63.214 & 13.266 & 12.980 \\
\hline & 4.0 & 37.697 & 58.983 & 212.491 & 58.846 & 8.983 & 8.612 \\
\hline & 6.0 & 25.812 & 53.318 & 276.000 & 53.412 & 3.318 & $3.178 \mathrm{~J}$ \\
\hline & 8.0 & 19.263 & $\$ 1.221$ & 321.075 & 51.371 & 1.221 & 1.137 \\
\hline & 10.0 & 15.083 & 50.449 & 355.421 & 50.625 & 0.449 & 0.391 \\
\hline & 12.0 & 12.096 & 50.165 & 383.600 & 50.456 & 0.165 & 0.222 \\
\hline & 14.0 & $9.810)$ & 50.061 & 404.506 & 50.261 & & \\
\hline & 16.0 & 7.998 & 50.022 & 422.314 & 50.229 & & \\
\hline & 18.0 & $6.535\} b$ & 50.008 & 436.847 & $50.220\}$ & & \\
\hline & 20.0 & 5.346 & 50.003 & 448.728 & 50.219 & & \\
\hline & 24.0 & $3.581)$ & 50.000 & 466.582 & $50.239)$ & & \\
\hline
\end{tabular}

${ }^{a} \hat{F(T)}=C_{1}(T)+(\hat{\beta})$ (trapezoidal area).

${ }^{b} \ln C_{1}=3.6915-0.100712 t(r=-0.99999)$ or $C_{1}=40.103 e^{-0.10 t}$, hence $\hat{\beta}=0.1$ and $\hat{A}_{2}=40.103$.

$c \hat{A}_{s}=$ average value of $F(T)$ for points used to estimate $\beta=50.234$.

$\ln \left[\hat{F(T)}-\hat{A}_{s}\right]=4.1756-0.5051 t(r=-1.0000)$ or $\hat{F}(T)-\hat{A}_{s}=65.076 e^{-0.5051 t}$, hence $\hat{I}_{1}=65.076$ and $\hat{Q}=0.5051$. Only the points which were randomly distributed about the apparent straight line were used-which explains the omission of the last two points.

Table II. Results of Simulation Example No. 1 Using Equations 15-19 Based on the Wagner-Nelson Method

\begin{tabular}{cccl}
\hline Parameter & Real value & Estimated value & Estimated value calculated with \\
\hline$C_{0}$ & 100 & 99.22 & Equation 16 \\
$\alpha$ & 0.5 & 0.5051 & See footnote $d$ of Table I \\
$\beta$ & 0.1 & 0.1 & See footnote $b$ of Table I \\
$k_{12}$ & 0.150 & 0.152 & Equation 19 \\
$k_{21}$ & 0.250 & 0.256 & Equation 17 \\
$k_{\text {el }}$ & 0.200 & 0.197 & Equation 18 \\
$k_{a}$ & 2.00 & 2.04 & Equation 15 \\
\hline
\end{tabular}


Applying equations 15-19 with the constants estimated by the WagnerNelson method gave the values shown in column 3 of Table II.

Using the "feathering" or "back-projection" technique, shown in Fig. 1 and Table III, the final equation 36 was obtained. Using equations 36-39 to obtain estimates of $C_{0}$ and $k_{21}$, respectively, and equations 18 and 19 to obtain estimates of $k_{\mathrm{el}}$ and $k_{12}$, respectively, this method gave the results

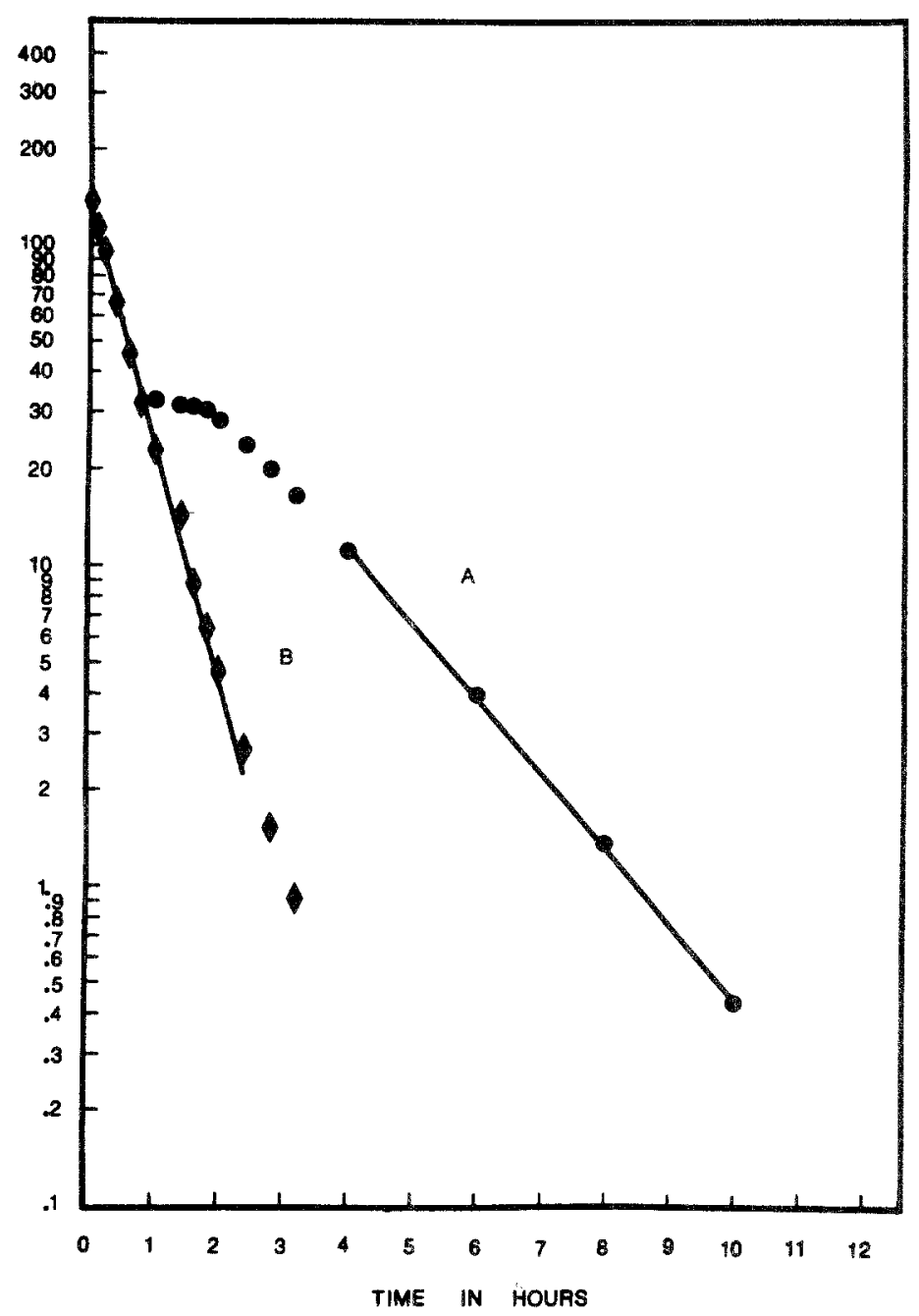

Fig. 1. "Feathering" method used in simulation example No. 1 to obtain equation 36. The line labeled " $A$ " is a plot of $R_{1}$, and the line labeled "B" is a plot of $R_{2}$. Numerical values and details are given in Table III. 
Table III. Estimation of Parameters for Simulation Example No. 1 by "Feathering" or "BackProjection"

\begin{tabular}{|c|c|c|c|c|c|}
\hline$t$ & $C_{1}$ & $40.103 e^{-0.100712 t}$ & $R_{1}{ }^{a}$ & $95.34 e^{-0.5364 t}$ & $R_{2}^{b}$ \\
\hline 0 & 0 & 40.103 & -40.103 & 95.34 & 135.44 \\
\hline 0.1 & 17.804 & 39.7010 & -21.8971 & 90.36 & 112.3 \\
\hline 0.2 & 31.775 & 39.3033 & -7.5283 & 85.64 & 93.17 \\
\hline 0.4 & 50.973 & 38.5196 & 12.4534 & 76.93 & 64.48 \\
\hline 0.6 & 61.921 & 37.7515 & 24.1695 & 69.10 & 44.93 \\
\hline 0.8 & 67.504 & 36.9987 & 30.5053 & 62.07 & 31.56 \\
\hline 1.0 & 69.641 & 36.2609 & 33.3801 & 55.76 & 22.38 \\
\hline 1.29726 & 69.063 & 35.1914 & 33.8716 & 47.54 & 13.67 \\
\hline 1.4 & 68.231 & 34.8291 & 31.4019 & 44.99 & 13.59 \\
\hline 1.6 & 66.075 & 34.1346 & 31.9404 & 40.42 & 8.48 \\
\hline 1.8 & 63.496 & 33.4539 & 30.0421 & 36.30 & 6.26 \\
\hline 2.0 & 60.726 & 32.7868 & 27.9392 & 32.61 & 4.67 \\
\hline 2.4 & 55.140 & 31.4923 & 23.6477 & 26.31 & $2.66)$ \\
\hline 2.8 & 49.929 & 30.2488 & 19.6802 & 21.23 & 1.55 \\
\hline 3.2 & 45.284 & 29.0545 & 16.2295 & 17.13 & 0.90 \\
\hline 4.0 & 37.697 & 26.8054 & 10.8916 & \multirow{3}{*}{\multicolumn{2}{|c|}{$\begin{array}{l}\ln R_{1}=4.5574-0.5364 t \\
(r=-0.9998)\end{array}$}} \\
\hline 6.0 & 25.812 & 21.9152 & 3.8968 & & \\
\hline 8.0 & 19.263 & 17.9171 & $1.3459\}$ & & \\
\hline $\begin{array}{l}10.0 \\
12.0\end{array}$ & $\begin{array}{l}15.083 \\
12.096\end{array}$ & $\begin{array}{l}14.6484 \\
11.9760\end{array}$ & $\begin{array}{l}0.4346 \\
0.1200\end{array}$ & \multicolumn{2}{|l|}{$R_{1}=95.34 e^{-0.5364 t}$} \\
\hline $\begin{array}{l}12.0 \\
14.0\end{array}$ & $\begin{array}{c}12.096 \\
9.810)\end{array}$ & & 0.12007 & \multicolumn{2}{|l|}{ Hence $\alpha=0.5364$} \\
\hline 16.0 & 7.998 & \multicolumn{4}{|c|}{$\ln C_{1}=3.6915-0.100712 t(r=-0.99999)$} \\
\hline 18.0 & $6.535\}$ & \multirow{2}{*}{\multicolumn{4}{|c|}{$C_{1}=40.103 e^{-0.100712 t}$}} \\
\hline 20.0 & 5.346 & & & & \\
\hline 24.0 & 3.581 & \multicolumn{4}{|c|}{ Hence $\hat{\beta}=0.100712$ and $A_{2}=40.103$} \\
\hline
\end{tabular}

${ }^{a} R_{1}=C_{1}-40.103 e^{-0.100712 t}$.

${ }^{b} R_{2}=95.34 e^{-0.5364 t}-R_{1}$.

${ }^{c} \operatorname{In} R_{2}=4.8354-1.651 t(r=-0.9988)$, hence $R_{2}=125.89 e^{-1.6510 t}$.

summarized in Table IV.

$$
C_{1}(t)=95.34 e^{-0.5364 t}+40.103 e^{-0.100712 t}-125.89 e^{-1.651 t}
$$

corresponding to

$$
C_{1}(t)=A_{1} e^{-\alpha t}+A_{2} e^{-\beta t}-A_{3} e^{-k_{a} t}
$$

where $A_{1}=95.34, A_{2}=40.103$, and $A_{3}=125.89$. Based on these equations, one obtains the estimates of $C_{0}$ and $k_{21}$ with equations 38 and 39:

$$
\begin{aligned}
C_{0} & =\frac{A_{1}\left(k_{a}-\alpha\right)+A_{2}\left(k_{a}-\beta\right)}{k_{a}} \\
k_{21} & =\frac{A_{1} \beta k_{a}+A_{2} \alpha k_{a}+A_{3} \alpha \beta}{A_{1}\left(k_{a}-\alpha\right)+A_{2}\left(k_{a}-\beta\right)}
\end{aligned}
$$


Table IV. Results of Simulation Example No. 1 Using Equations 36-39 Based on "Feathering" or "Back-Projection"

\begin{tabular}{cccl}
\hline Parameter & Real value & Estimated value & Estimated value calculated with \\
\hline$C_{0}$ & 100 & 102.0 & Equation 38 \\
$\alpha$ & 0.5 & 0.5364 & See Table III and Fig. 1 \\
$\beta$ & 0.1 & 0.1 & See Table III and Fig. 1 \\
$k_{12}$ & 0.150 & 0.169 & Equation 19 \\
$k_{21}$ & 0.250 & 0.264 & Equation 39 \\
$k_{\text {el }}$ & 0.200 & 0.203 & Equation 18 \\
$k_{a}$ & 2.00 & 1.65 & See Table III and Fig. I \\
\hline
\end{tabular}

It is interesting that the estimated parameters shown in Table II, obtained by the Wagner-Nelson method (at least in this case), are closer to the real values than those obtained via the usual "feathering" or "back-projection" technique shown in Table IV. This is particularly true for the estimate of $k_{a}$, where the Wagner-Nelson method gave almost the exact value whereas the "feathering" method gave an estimate that was $17.5 \%$ low.

\section{Simulation Example No. 2 for Abrupt Cessation of Absorption}

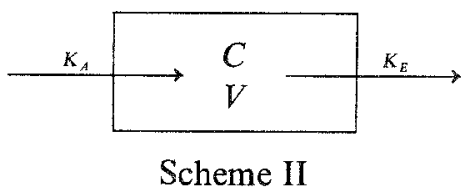

In Scheme II, $K_{A}$ and $K_{E}$ are the first-order rate constants for absorption and elimination, respectively. Assume that the model shown in Scheme II applies, and that $A_{0} / V=100$ units for one dosage form and 50 units for another dosage form. Assume that $K_{A}=1.0455 \mathrm{hr}^{-1}$ and $K_{E}=0.17425$ $\mathrm{hr}^{-1}$. Then we can write equations 40 and 41 for the two cases:

$$
\begin{aligned}
& A_{T} / V=100\left(1-e^{-1.0455 t}\right) \\
& A_{T} / V=50\left(1-e^{-1.0455 t}\right)
\end{aligned}
$$

Let us assume that absorption ceases when $70 \%$ of the dose is absorbed. One can then readily calculate that this would occur at $1.15158 \mathrm{hr}$, and at this time the value of $C$ would be 62.183 units when $A_{0} / V=100$ units. In the interval $0 \leq t \leq 1.15158 \mathrm{hr}, C$, in the 100 -unit case, would be given by equation 42 :

$$
C=120\left(e^{-0.17425 t}-e^{-1.0455 t}\right)
$$


In the interval $t \geq 1.15158 \mathrm{hr}, C$ would be given by equation 43 in the 100 unit case:

$$
C=(62.183) e^{-0.17425(t-1.15158)}
$$

$C ; t$ data were generated by use of equations 42 and 43 , and analogous equations for the 50-unit case, for various values of $t$ up to $15 \mathrm{hr}$. Then the Wagner-Nelson method was applied to the simulated data. The results are shown in Table $\mathrm{V}$ and the plots of $C_{T}+K_{E} \int_{0}^{T} C \cdot d t$ vs. $T$ are shown in Fig. 2. From Table V, one can see that the values of the function, $F(T)$, are identical, within the error of the trapezoidal rule and round-off, to the exact values of $A_{T} / V$ obtained from equations 40 and 41 . In Fig. 2, it should be noted that the asymptotes are abruptly reached, rather than approached slowly as would be expected for a completed first-order reaction. Thus, in this case, the asymptotes of approximately 70 and 35 are not the same as the

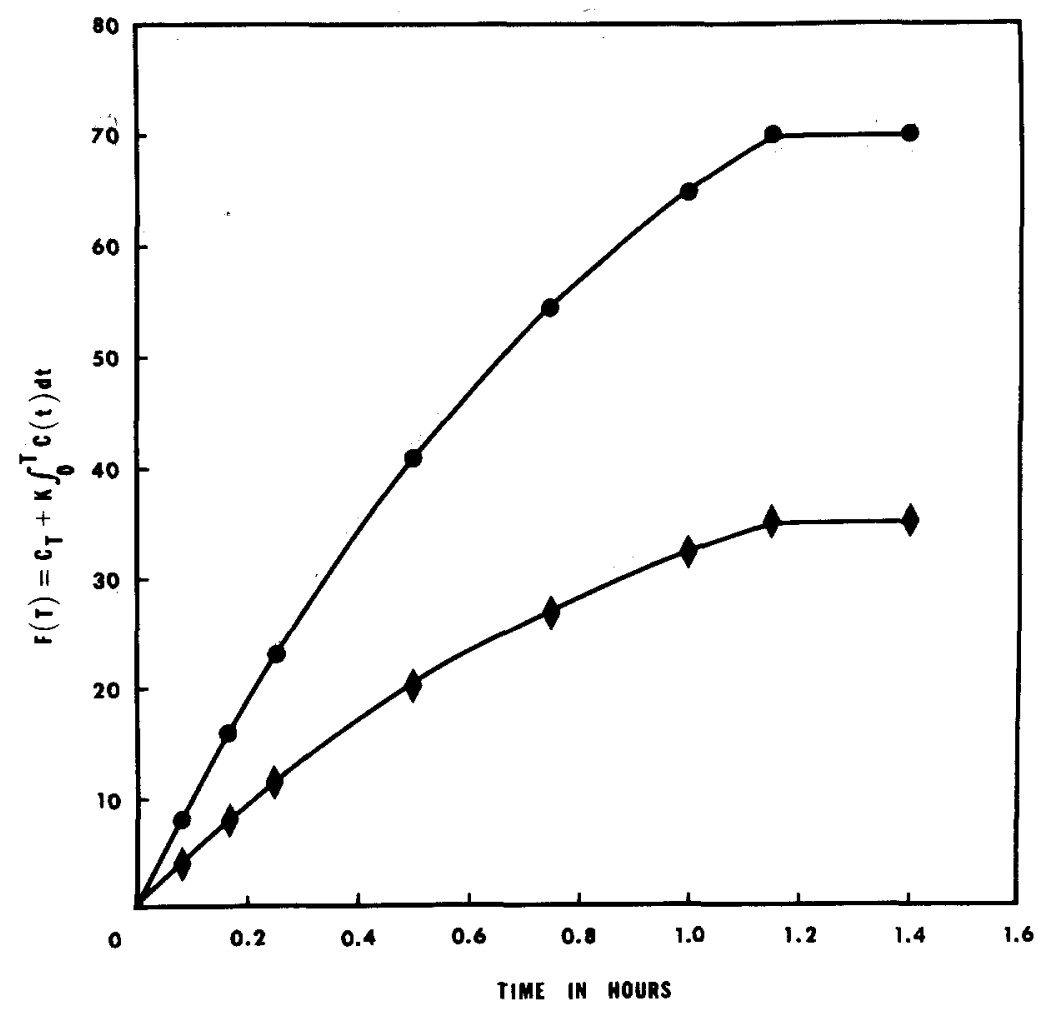

Fig. 2. Application of the Wagner-Nelson method in simulation example No. 2. See details in Table V. 


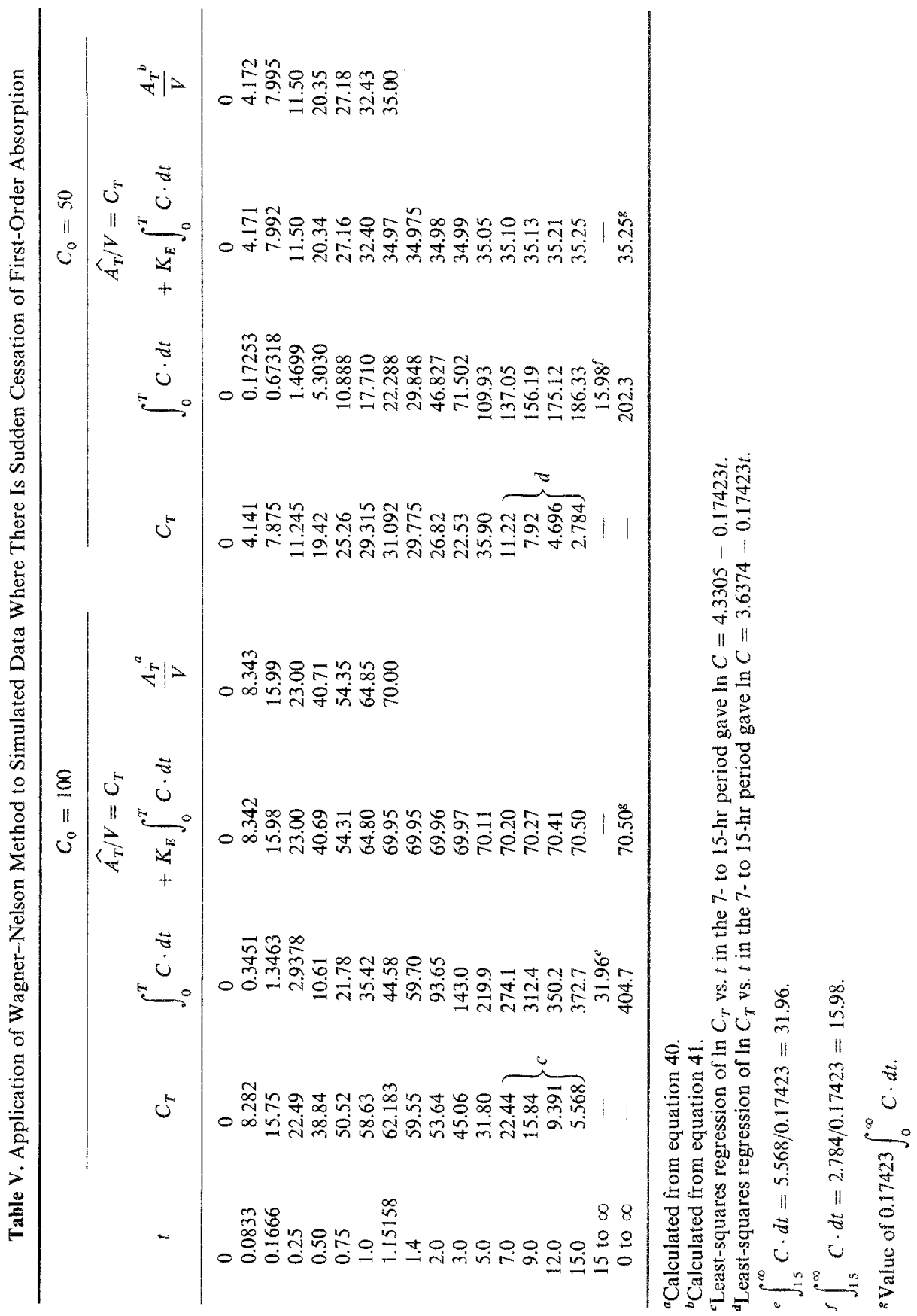


Table VI. Application of the Guggenheim Method (7) to the $\widehat{A_{T}} / V$ Values Shown in Table $\mathrm{V}$

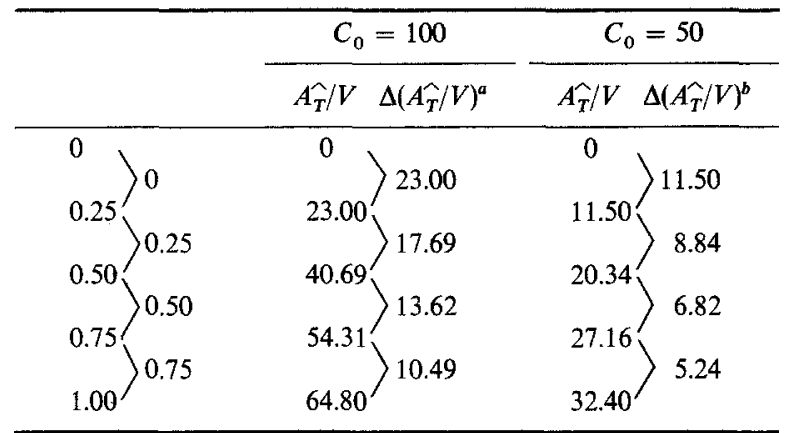

${ }^{a}$ Least-squares regression of $\ln \left[\Delta\left(\widehat{A_{T}} / V\right)\right]$ vs. $t_{1}$ gave

$$
\ln \left[\Delta\left(\hat{A_{T}} / V\right)\right]=3.1351-1.0467 t_{1}
$$

or $\Delta\left(\hat{A_{T}} / V\right)=22.99 e^{-1.0467 t_{1}}$, hence $\widehat{k_{a}}=1.0467$ (actual value was 1.0455$)$. Hence $\hat{C_{0}}=22.99 /\left[1-e^{-(1.0467)(0.25)}\right]=99.9$ (actual value was 100 ).

${ }^{b}$ Least-squares gave $\ln \left[\Delta\left(\widehat{A_{T}} / V\right)\right]=2.4421-1.0470 t_{1}$ or

$$
\Delta\left(\widehat{A_{T}} / V\right)=11.50 e^{-1.047 t_{1}}
$$

hence $\widehat{k_{a}}=1.047$ (actual value was 1.0455). Hence

$$
\widehat{C_{0}}=11.50 /\left[1-e^{-(1.047)(0.25)}\right]=49.9
$$

(actual value was 50 ).

coefficients 100 and 50 of equations 40 and 41 , respectively. When one does not know the actual asymptote of a first-order process, one can calculate the rate constant by the method of Guggenheim (7). Application of this method, shown in Table VI, to the $A_{T} / V$ values at $0,0.25,0.5,0.75$, and $1.0 \mathrm{hr}$ gave estimated $K_{A}$ values of 1.0467 and $1.047 \mathrm{hr}^{-1}$, which are essentially identical to the actual value of $1.0455^{-1}$. Thus the Wagner-Nelson method, followed by appropriate analysis. of the $A_{T} / V$ vs. $T$ plot, gives the correct estimate of the first-order absorption rate constant in the case where absorption abruptly ceases and availability is less than $100 \%$.

Ignoring the shape of the plots shown in Fig. 2, and calculating values of percent remaining unabsorbed by using the asymptotes 70.50 and 35.25 in the denominators when the percentages absorbed are calculated, leads to the same values for both sets of data. These percentages are plotted in Fig. 3. It is obvious that the points form a curved line and that one should not estimate a first-order rate constant from such data. This type of error, where the wrong asymptote is used in estimating a first-order rate constant, was discussed by Wagner (8) in 1963 . Thus Perrier and Gibaldi (4) are correct 


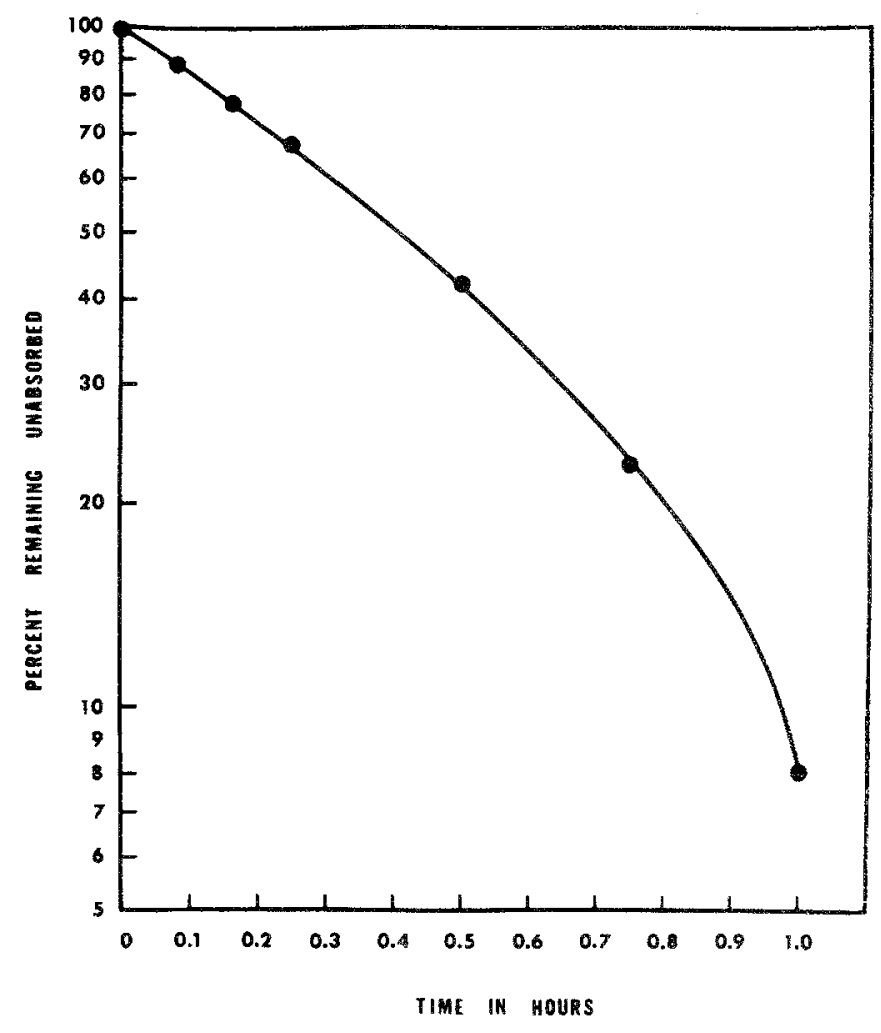

Fig. 3. Plot of percent remaining unabsorbed on logarithmic scale against time when wrong asymptote is used in simulation example No. 2.

that if one makes this error biased first-order absorption rate constants are obtained. Use of the Guggenheim method, or any alternative method not involving the asymptote, circumvents the problem.

\section{DISCUSSION}

The Wagner-Nelson (1) and Loo-Riegelman (6) methods were derived for models not involving competing reactions at the absorption site. The functions obtained by these methods are exactly equal to $A_{T} / V$ or $A_{T} / V_{1}$, respectively, in the case where there is no competing reaction at the absorption site. The functions obtained by these methods are also exactly equal to $A_{T} / V$ or $A_{T} / V_{1}$ respectively, in the case where there is first-order absorption which abruptly ceases after some time and before the entire dose is absorbed. It was shown that in the latter case the correct absorption rate constant may 
be estimated by applying the Guggenheim method to the $A_{T} / V$ values rather than the usual $\sigma$-minus plot. It must be remembered that the Guggenheim method requires that the $A_{T} / V$ values be obtained at equally spaced time intervals during the major portion of the absorption phase.

A competing reaction at the absorption site due to chemical degradation of a drug in the gastrointestinal fluids, such as discussed by Notari et al. (3), would cause considerable problems in the interpretation of absorption plots obtained by both the Loo-Riegelman (6) and Wagner-Nelson (1) methods. One of the models discussed by Perrier and Gibaldi (4) is shown as Scheme III :

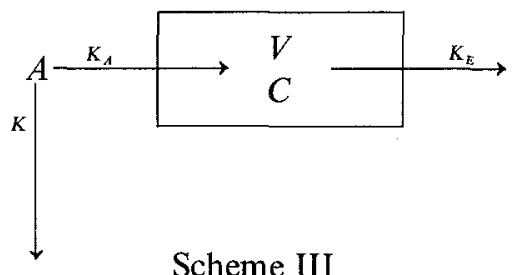

It should be noted that this model assumes that every molecule of $A$ is in solution at $t=0$, or, after some lag time, $t_{0}$, and that all are simultaneously acted on by two first-order processes with rate constants $K_{A}$ and $K$. Hence such a model applies to the chemical degradation case as discussed by Notari et al. (3). However, the author does not believe that the model shown in Scheme III can be applied to the case where there is an "absorption window" in the upper gastrointestinal tract and the rate constant " $K$ " refers to gastrointestinal transit of the solution of the drug, such as discussed by Perrier and Gibaldi (4). When the rate constant " $K$ " describes the physical process of removal of drug from the absorption site by gastrointestinal transit, we should not write a single model such as shown in Scheme III, but rather sequential models as shown in Schemes IVa and IVb:

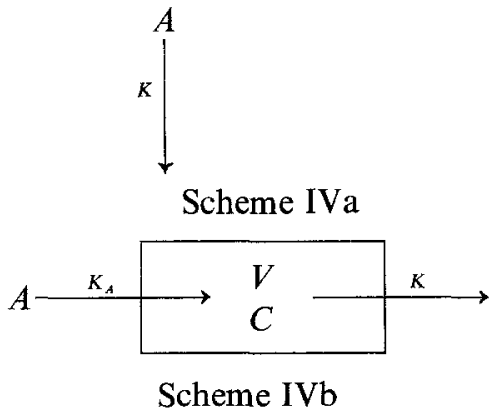

Before the drug reaches the "window" and past the "window," the model shown in Scheme IVa applies. In the window, the model shown in Scheme IVb 
applies when the disposition model is the one-compartment open model. In the window, the initial driving force $\left(A_{0}\right)$ would be the amount of drug which has reached the solution state prior to the window. Also, since firstorder kinetics are independent of the initial amount or concentration, the same $K_{A}$ will be measured for a fixed window but different $A_{0}$ values. The observed $K_{A}$ will depend on the size of the window (as it always does), since $K_{A}$ incorporates the area of the membrane. Thus the problem posed by Perrier and Gibaldi (4) may be overcome by using the consecutive model approach.

\section{APPENDIX}

Equation 7 may also be written as equation $7 \mathrm{a}$.

$$
\begin{aligned}
F(T)=C_{1}(T)+\beta \int_{0}^{T} C_{1}(t) d t= & C_{0}\left[\frac{k_{21}}{\alpha}+\frac{1}{\alpha\left(\alpha-k_{a}\right)}\left\{\alpha\left(k_{a}-k_{21}\right) e^{-k_{a} T}\right.\right. \\
& \left.\left.-k_{a}\left(\alpha-k_{21}\right) e^{-\alpha T}\right\}\right]
\end{aligned}
$$

In the following, alternative equations (with numbers followed by "a") replace previous equations with the same numbers (but without the "a") for the case when $\alpha>k_{a}>\beta$.

$$
F(T)=A_{s}+I_{1} e^{-k_{a} t}-I_{2} e^{-\alpha T}
$$

where

$$
I_{1}=C_{0}\left(k_{a}-k_{21}\right) /\left(\alpha-k_{a}\right)
$$

and

$$
I_{2}=C_{0} k_{a}\left(\alpha-k_{21}\right) / \alpha\left(\alpha-k_{a}\right)
$$

After combining equations 8 and $10 \mathrm{a}$, one obtains

$$
k_{a}=\alpha\left(I_{1}+A_{s}\right) /\left(C_{0}+I_{1}\right)
$$

By equating the right-hand sides of equations 14 and $15 \mathrm{a}$, one obtains

$$
C_{0}=\frac{\beta A_{2}(\alpha-\beta) I_{1}+\left\{\alpha^{2} A_{s}-\alpha(\alpha-\beta) A_{2}\right\}\left\{I_{1}+A_{s}\right\}}{\alpha \beta\left(I_{1}+A_{s}\right)-\beta A_{2}(\alpha-\beta)}
$$

Equations 8, 12-14, and 17-19 are valid for this alternative case.

Example: Let $k_{a}=0.5, \alpha=2, \beta=0.1, k_{21}=0.25$, and $C_{0}=100$. Substitution into equation 6 gives equation $34 \mathrm{a}$ :

$$
C_{1}(t)=20.8333 e^{-0.5 t}+9.868421 e^{-0.1 t}-30.7018 e^{-2 t}
$$


Substitution into equations $8,10 \mathrm{a}$, and $11 \mathrm{a}$ gives equation $9 \mathrm{~b}$ from $9 \mathrm{a}$ :

$$
F(T)=12.5+16.6666 e^{-0.5 T}-29.1666 e^{-2 T}
$$

Thus $A_{2}=9.868421, A_{s}=12.5$, and $I_{1}=16.6666$. Substitution of these values into equation $16 \mathrm{a}$ yields the expected value of 100 for $C_{0}$. It should be noted that in applying the alternative equations above $k_{a}$ replaces $\alpha$ in equation 20 .

\section{REFERENCES}

1. J. G. Wagner and E. Nelson. Percent absorbed time plots derived from blood level and/or urinary excretion data. J. Pharm. Sci. 52:610-611 (1963).

2. J. G. Wagner and E. Nelson. The kinetic analysis of blood levels and urinary excretion in the absorptive phase after single doses of drug. J. Pharm. Sci. 53:1392-1403 (1964).

3. R. E. Notari, J. L. DeYoung, and R. H. Reuning. Effect of parallel first-order drug loss from site of administration on calculated values for absorption rate constants. J. Pharm. Sci. 61:135-138 (1972).

4. D. Perrier and M. Gibaldi. Calculation of absorption rate constants for drugs with incomplete availability. J. Pharm. Sci. 62:225-228 (1973).

5. L. J. Leeson and H. Weintraub. Calculating absorption rate constants for drugs with incomplete bioavailability. J. Pharm. Sci. 62:1936-1941 (1973).

6. J. C. K. Loo and S. Riegelman. New method for calculating the intrinsic absorption rate of drugs. J. Pharm. Sci. 57:918-928 (1968).

7. E. A. Guggenheim. On the determination of the velocity constant of a unimolecular reaction. Phil. Mag. 2:538-543 (1926).

8. J. G. Wagner. Some possible errors in the plotting and interpretation of semilogarithmic plots of blood level and urinary excretion data. J. Pharm. Sci. 52: 1097-1101 (1963). 